\title{
Comparative Study of Pottery Industry in Zeh-Klout Historical Period in Roudbar-Kerman with Its Neighbouring Cultures Based on the New Archaeological Evidences
}

\author{
Samira Sheikhakbari ${ }^{1}$, Hossein Sarhaddi-Dadian ${ }^{2,}$, , Saeed Amirhajloo ${ }^{3}$, Ali Daneshi ${ }^{4}$ \\ ${ }^{1}$ Archaeologist, Independent Researcher, Kerman, Iran \\ ${ }^{2}$ Department of Archaeology and Archaeological Research Center, University of Zabol, Zabol, Iran \\ ${ }^{3}$ Department of Archaeology, University of Jiroft, Jiroft, Iran \\ ${ }^{4}$ Jiroft Archaeological Research Complex, Jiroft, Iran
}

\section{Email address:}

S.sheikhakbary2004@yahoo.com (S. Sheikhakbari), Hossein.Sarhaddi@gmail.com (H. Sarhaddi-Dadian), saeed.hajloo@gmail.com (S. Amirhajloo), alidaneshi222@yahoo.com (A. Daneshi)

\section{To cite this article:}

Samira Sheikhakbari, Hossein Sarhaddi-Dadian, Saeed Amirhajloo, Ali Daneshi. Comparative Study of Pottery Industry in Zeh-Klout Historical Period in Roudbar-Kerman with Its Neighbouring Cultures Based on the New Archaeological Evidences. International Journal of Archaeology. Vol. 3, No. 3, 2015, pp. 22-32. doi: 10.11648/j.ija.20150303.11

\begin{abstract}
Zeh-Kalout is located at western Jazmouriyan in Roudbar, Kerman Province. Due to several reasons such as suitable environmental condition appropriate climate, and rich water supplies this area has been a favorable location for human settlement from pre-historic times through contemporary period. Recorded items from Zeh-Kalout depict its strategic commercial and economic significance in various periods. The potteries are one of the most important cultural materials that have been found in the following research. Comparing this pottery with similar items reveals the relationships between this area and the surrounding Cultures including Boushehr, Fars, Sistan, and the eastern satrapies of Pakistan, Afghanistan, Persian Gulf, and Oman. The Present research describes, classifies, and morphologies the historical Potteries of Zeh-Kalout based on the cultural discoveries(Pottery), and archeological document historical Pottery work of Zeh-Kalout are generally plain and without patterns. They are made from a mineral combination of sand. They are divided into three groups: bowls, urns, and jars and has a long relationship with adjacent cultures.
\end{abstract}

Keywords: Archaeological Survey, Zeh-Kalout, Halil Rood Region, Historical Periods, Morphology of Pottery, Southern Kerman, Iran, Adjacent Cultures

\section{Introduction}

According to the remaining historical writings of Jazmoryan area in Achaemenid period with various names (Makn, Gadrou, Ecofajia) which are parts of this area, it shows that: Darioush in his charter states that, Yaka wood, MishMakn are transferred from Gedara to Kerman.

Herodotus speaks about twenty Satraps, the Achaemenid Empire which includes Gedrozia Makran. The ancient path of Pars and Lorestan had passed between the Send valley through Jiroft, Roudbar and Bampur. There were also shielding castles, infrastructures and pathes. In second volume of Herodotus's book, page 485 Ramilson publication, he says a part of this area had been under domination of 17th Darioush [1].

According to Eristoboul report from Alexander travel in
Arian book, Alexander had passed from Makoran, Gedrusia and Ecofajia on his way from India to pars. He believes that it's the path of Semiramis the legend Queen of Babel and Cyrus the Great the son of Cambyses I and most of their followers had died in its difficult path.

Alexander also dealt to the lack of water and food, torrential rains and winds and severe storms in this gateway. He had lost his way many times and his people has died due to the thirsty and hunger and hot weather. It took 60 days to him to arrive to Poura the capital of Gedrusia from Uria. The Alexander army could finally rest in this city. In this passage, his men destroyed the Temple and the old graves and they had dealt with indigenous people, and it's been said that, Alexander punished and executed violators [2]

With continuing development of the Achaemenid period and the successors of Alexander, canal and aqueducts, 
operation of water resources has improved and dams are created in the valleys of the mountains and the rivers. According to credible resources in Parthian era, this region was a part of Kushan's territory and it was a commercial link between Iran and India. The maritime trade and the trade of silk fabrics and dishes from China, herbs and medicinal plants from India, slaves from Africa, as well as Iranian-made agricultural products enjoyed a boom in Parthian era. Parthian government had completed the previous governments' efforts (Elamite and Achaemenid) for security of business convoy [3].

According to the Zarathustra-based inscriptions, Makoran was one of the thirty-three of the cities in the third century AD in Shapur era. It was located between Kerman, Thurston, Sikeston and India. During the Sassanian era, the Gedrusia or Macron was the best developed, due to the Flourished trade and improvement in resource utilization and creating dams over the revers, utilizing the great labor in agricultural production as well as Thriving silk fabric industry and metallurgy, traveling the commercial convoys. Gedrusia or Macron is one of the ten productive lands in Iran, in Gobad Geography. During the Sassanian era, some of the Tribes living in northwest and western of Iran were immigrated to Southeast of Iran and they were called "Balouch" [4].

\section{The Geographical Location of the Area Under Study}

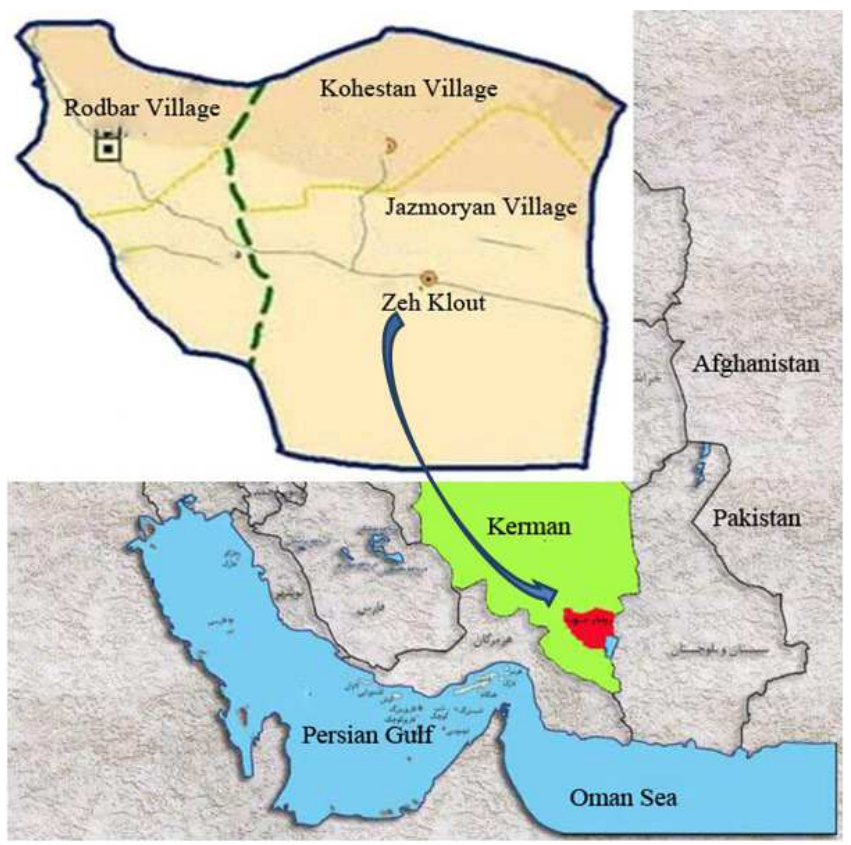

Fig. 1. The location of Study.

Zeh-Klout is located $67 \mathrm{~km}$ South-East of Islamabad (south of Roudbar), (Geographical culture of villages in Kerman province[5]. The boundaries of this city meet anberAbad in North, Iranshahr in south, Bam in east and Kahnooj and Ghale Ganj in west (Fig 1). Zeh-Klout natural position is plain, but the mountains and heights can be seen around the village. Doukam seasonal river passes from the West of this village. This survey was conducted in 1391 in winter in Provincial funding framework, with the authorization of the Institute of Archaeology in order to, Register, introduce and analyze the sites and monuments and archaeological sites and producing archaeological atlas produced in Zeh-Klout, Jazmoryan, and also for evaluating the relationship between archaeological sites and neighboring cultures.

\section{Research Methodology}

This Research is conducted in two methods: (a) library method, and (b) field method. In the first method, we have tried to collect all data and available resources about this area in Historical and geographical documents and in second method, we tried to collect Samples indicators of cultural material by checking the site and then describe and classify obtained Technical Specifications and potteries (bowls, jugs, etc.) from this Zeh-Klout. After typological Comparison and analyzing the obtained result in tables, the map and plan are described and the compared sample plans are indicated in illustrative tables with reference samples.

\section{Archaeological Background}

The first Systematic archaeological studies are conducted by Stien an English, Hungarian-born archaeologist in 1936 [6]. In 1943 Harrison from Britain's Royal Geographical Forum, visited this site with the goal of identifying the Land and recognizing the oil resources of Baluchestan and he prepared a Comprehensive geological map [7]. There are also some surveys conducted by Sarfaraz and Babak Rad in Kerman [8], and also Gari Hume in Ladiz [9]. Sajjadi also evaluated about three thousand kilometers of this area [10]. Another step of archaeological excavations and surveys began in 1380. Hamideh Choubak has conducted Rescue excavations of plundered area of Rig Anbar and Konar Sandal in 1380and also identified Works related to the Bronze period [11]. Majid-Zadeh has evaluated the Konar Sandal in 1381-1382 [12]. The predicted surveys of Daghianous Site (the old city of Jiroft) started in 1381 and its second part continued in 1382-1383.[3]. Also Vali-Allah Dehghan evaluated a part of Roudbar from Bahador Abad in $25 \mathrm{~km}$ North-West of Islamabad - to Mir Meghdad village At a distance of approximately 45 kilometers SouthEast - with a triangle shape and the area of 700 Square Meter [13].

69 sites with cultural heritage were identified and registered in evaluating the Zeh-Klout. Its oldest site is related to Fourth millennium before Christ and the newest site is related to two Cemeteries in Late Islamic period. Historical periods are the most important residential periods in Zeh-klout. 51 sites with cultural signs from historical periods and comparing them with previous eras indicates the Growth and prosperity of the region and the settlement and population growth in Zeh-Klout. The numbers of 
cemeteries are more than settlement. And it's maybe related to the Migration and nomadic life on the south of Kerman. There are still the Migration and nomadic life on the south of Kerman too [14], and the sites are still located on The ridges and rivers. In general Zeh-Klout settlement can be divided into Seasonal, permanent and defensive installations (castles).

\section{Potteries of Zeh-Klout Historical Period}

Most cultural heritage derived from the study of Zeh-Klout Jazmoryan is the Potteries collected from hills and sites. Introducing the collection of potteries in this period through classifying and comparing them with other areas in Iran is a fundamental step for better understanding of historical periods in this region. The purpose of this study is to introduce and classify and Typology the potteries in ZehKlout. By describing the potteries obtained from surveys, it helps us to recognize the cultural, economic and commercial status of people in Zeh-Klout.

\section{Potteries of Achaemenid Period}

In fact, pottery industry had an applicable nature in Achaemenid period and it wasn't used for showing the craftsmen's art. It's because of the absence of luxury wares in this era. It can be also seen in Achaemenid capitals [15]. In fact the metal and stone dishes provided an appropriate context for showing the craftsmen's art and potteries were just used as the applicable dishes [16].

The pottery art in Achaemenid period shows the Continuation of the first millennium potteries, Potters focused on a kind of simple designed potteries in this era [17]. The most of evaluations and researches are related to Pre-Islamic history. The tomb Kharg [18], and Yahya hill [19], are the areas with potteries from Achaemenid period. Besides, some of the potteries in Achaemenid period are similar to the potteries form other regions in Iran such as Dahane- Gholaman, Takhte Jamshid [20], and Pasargad [21]. The Potteries of Achaemenid period Zeh-Klout are simple and without any design and only have a castle with Black vertical lines on the edge and another part with the Horizontal line on the edge [Tables. 1,3,5,7,9,11 and 13].

Table 1. Comparative Table of Potteries of Qaleh-i Soniya Gazbor.

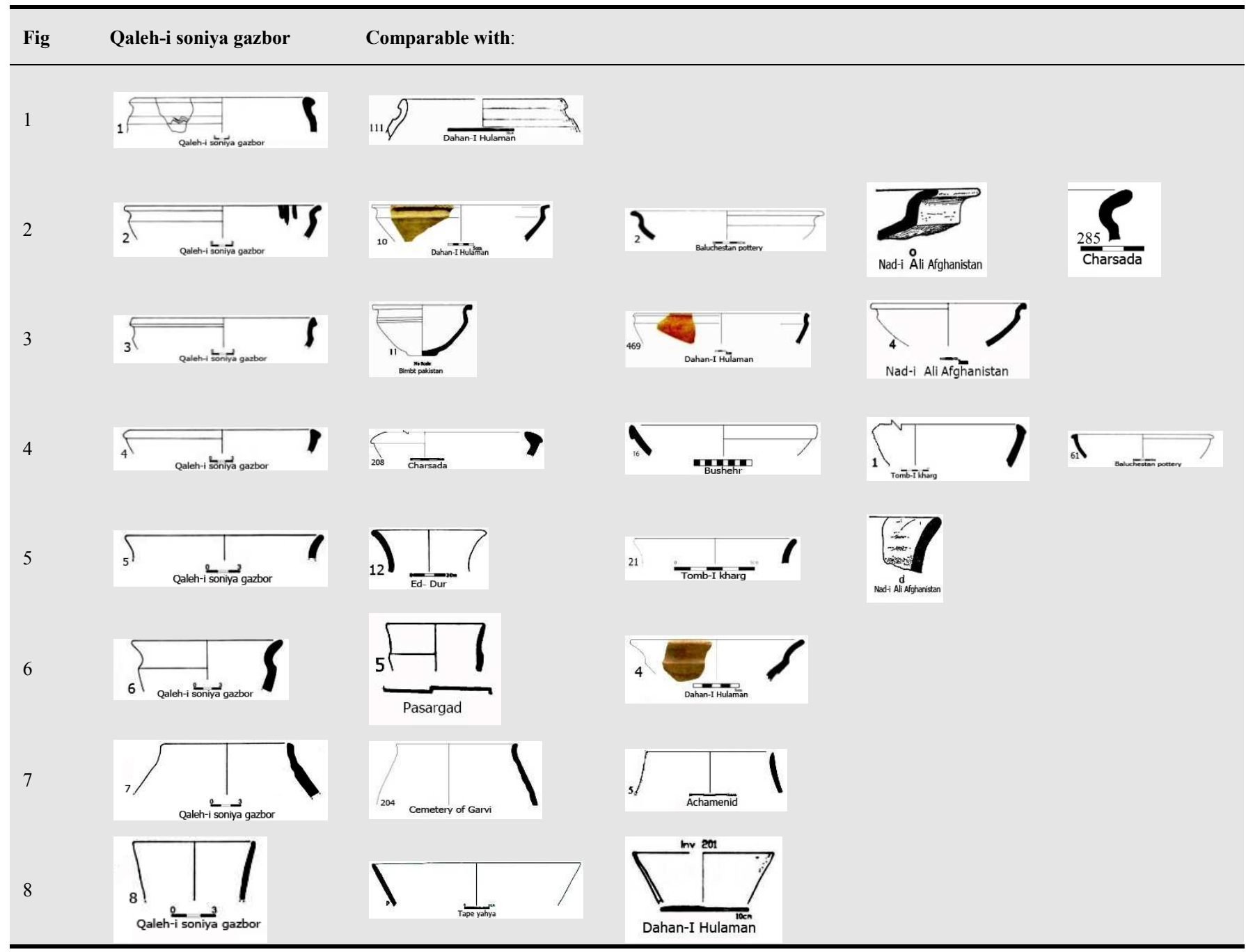


Table 2. Specification Table of pottery, Qaleh-i Soniya Gazbor.

\begin{tabular}{|c|c|c|c|c|}
\hline Site & Fig & $\begin{array}{l}\text { Specification: 1(Type)‘2.( Color oftemper) ‘3 (Temper) ‘ } \\
\text { 4(decoration)،5(Weyofprodoction) ‘6(Weyoffiring)‘ } \\
\text { 7.(slip)‘8(Dia- rim)،9(Dia- base). }\end{array}$ & Comparable with: & Refrence \\
\hline \multirow[t]{2}{*}{$\begin{array}{l}\text { Qaleh-i Soniya } \\
\text { gazbor }\end{array}$} & \multirow[t]{2}{*}{1} & \multirow[t]{2}{*}{$\begin{array}{l}\text { 1. Rim` 2. Brick- red‘ 3،grit and sand‘4.incised decoration‘5. } \\
\text { Wheel made ، 6.perfect ‘7. Brick- red ، } 8 \text {.(34) ‘9.(-). }\end{array}$} & Dahane Gholaman & $\begin{array}{l}\text { Harrassowitz 2010, fig 8: } \\
111\end{array}$ \\
\hline & & & Dahane Gholaman & Zehbari, 2012, fig 9:10 \\
\hline \multirow{3}{*}{$\begin{array}{l}\text { Qaleh-i Soniya } \\
\text { gazbor }\end{array}$} & \multirow{3}{*}{2} & \multirow{3}{*}{ 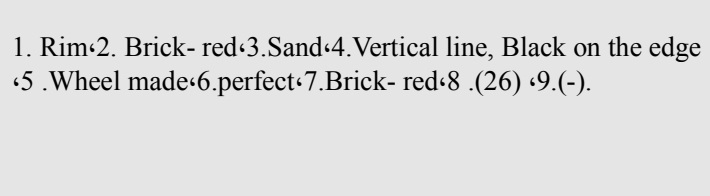 } & Baluchestan pottery & Alizade,1391,pl 1,fig2 \\
\hline & & & $\begin{array}{l}\text { Nad-i Ali in } \\
\text { Afghanistan }\end{array}$ & $\begin{array}{l}\text { Dales 1977, } \\
\text { fig 17: o }\end{array}$ \\
\hline & & & Charsada & $\begin{array}{l}\text { Wheeler 1962, fig } \\
32: 285\end{array}$ \\
\hline \multirow{3}{*}{$\begin{array}{l}\text { Qaleh-i Soniya } \\
\text { gazbor }\end{array}$} & \multirow{3}{*}{3} & \multirow{3}{*}{$\begin{array}{l}\text { 1. Rim‘2.Brick-red‘3.Sand‘4‘(-).5. Wheel made‘6. } \\
\text { Perfect‘7.Buff‘8.(24)، 9.(-). }\end{array}$} & Blmbat Pakistan & Dittman, 1984, fig 7:11 \\
\hline & & & Dahane Gholaman & Zehbari, 1391؛ fig 469 \\
\hline & & & $\begin{array}{l}\text { Nad-i Ali in } \\
\text { Afghanistan }\end{array}$ & Dales, 1977, pl.20, no 4 \\
\hline \multirow{6}{*}{$\begin{array}{l}\text { Qaleh-i Soniya } \\
\text { gazbor }\end{array}$} & \multirow{6}{*}{4} & \multirow{6}{*}{$\begin{array}{l}\text { 1. Rim`2.Brick-red‘3.sand‘4،(-).5. Wheel } \\
\text { made‘6 .Perfect‘7.Brick-red، } 8 \text {.(23) ‘9.(-). }\end{array}$} & Sohar & $\begin{array}{l}\text { Kervran, Heibert, 1991, fig } \\
4: 13\end{array}$ \\
\hline & & & Charsada & wheeller 1962 ; fig $27: 208$ \\
\hline & & & Bushehr & $\begin{array}{l}\text { Carter and challis and } \\
\text { priestman and tofighian } \\
\text { 2004, Fig 13:16 }\end{array}$ \\
\hline & & & Pasargad & Stronach, 1379؛ fig 12:108 \\
\hline & & & Tomb-i Kharg & Shahsavari, 1388, pl 5-4: 1 \\
\hline & & & Baluchestan pottery & $\begin{array}{l}\text { Alizade, 1391, } \\
\text { pl 13,fig61 }\end{array}$ \\
\hline \multirow{3}{*}{$\begin{array}{l}\text { Qaleh-i Soniya } \\
\text { gazbor }\end{array}$} & \multirow{3}{*}{5} & \multirow{3}{*}{$\begin{array}{l}\text { 1. Rim‘2.Brick-red‘3.Sand،4 Black line on the edge } 5 \text {. Wheel } \\
\text { made ،6.Perfect‘7.Buff‘8.(17) ‘9.(-). }\end{array}$} & Ed- dur & Iecomte. 1993, fig 9:12 \\
\hline & & & Tomb-i Kharg & $\begin{array}{l}\text { Shahsavari, } 1388, \text { pl 12- } \\
4: 21\end{array}$ \\
\hline & & & $\begin{array}{l}\text { Nad-i Ali in } \\
\text { Afghanistan }\end{array}$ & $\begin{array}{l}\text { Dales 1977, } \\
\text { fig 16: d }\end{array}$ \\
\hline \multirow{2}{*}{$\begin{array}{l}\text { Qaleh-i Soniya } \\
\text { gazbor }\end{array}$} & \multirow{2}{*}{6} & \multirow{2}{*}{$\begin{array}{l}\text { 1. Rim‘2.Brick-red‘3.Sand‘4،(-).5. Wheelmade ، } \\
\text { Imperfect.6،7.Buff ‘8. (16) ‘9.(-). }\end{array}$} & Pasargad & Stronach, 1379؛ fig 5:106 \\
\hline & & & Dahane Gholaman & Zehbari,2012, pl 8, fig 4 \\
\hline \multirow{2}{*}{$\begin{array}{l}\text { Qaleh-i Soniya } \\
\text { gazbor }\end{array}$} & \multirow{2}{*}{7} & \multirow{2}{*}{$\begin{array}{l}\text { 1. Rim‘2.Brick-red‘3.Sand‘4).-‘(5. Wheel } \\
\text { made‘6.Imperfect‘7 .Buff॰8 .(12) ‘9.(-). }\end{array}$} & Cemetery of Garvi & $\begin{array}{l}\text { Sarlak,1387, season1, fig: } \\
204\end{array}$ \\
\hline & & & Achamenid & Ataee, $1388, \mathrm{pl} 12: 3$ \\
\hline \multirow{2}{*}{$\begin{array}{l}\text { Qaleh-i Soniya } \\
\text { gazbor }\end{array}$} & \multirow{2}{*}{8} & \multirow{2}{*}{$\begin{array}{l}\text { 1. Rim‘2.Brick-red‘3.Sand‘4،(-).5. Wheel made، } \\
\text { 6.Perfect‘7.Buff‘8 .(8) ‘9.(-). }\end{array}$} & Tepe Yahya & $\begin{array}{l}\text { Lamberg-Karlovsky } 1967- \\
\text { 1969, fig:15.IV:A }\end{array}$ \\
\hline & & & Dahane Gholaman & $\begin{array}{l}\text { Harrassowitz 2010, fig 7: } \\
201\end{array}$ \\
\hline
\end{tabular}

Table 3. Comparative Table of Potteries of Sar Tape Lakkoh.

Fig Sar Tape Lakkoh Comparable with:


Table 5. Comparative Table of Potteries of Cemetery of Gavcharan II.

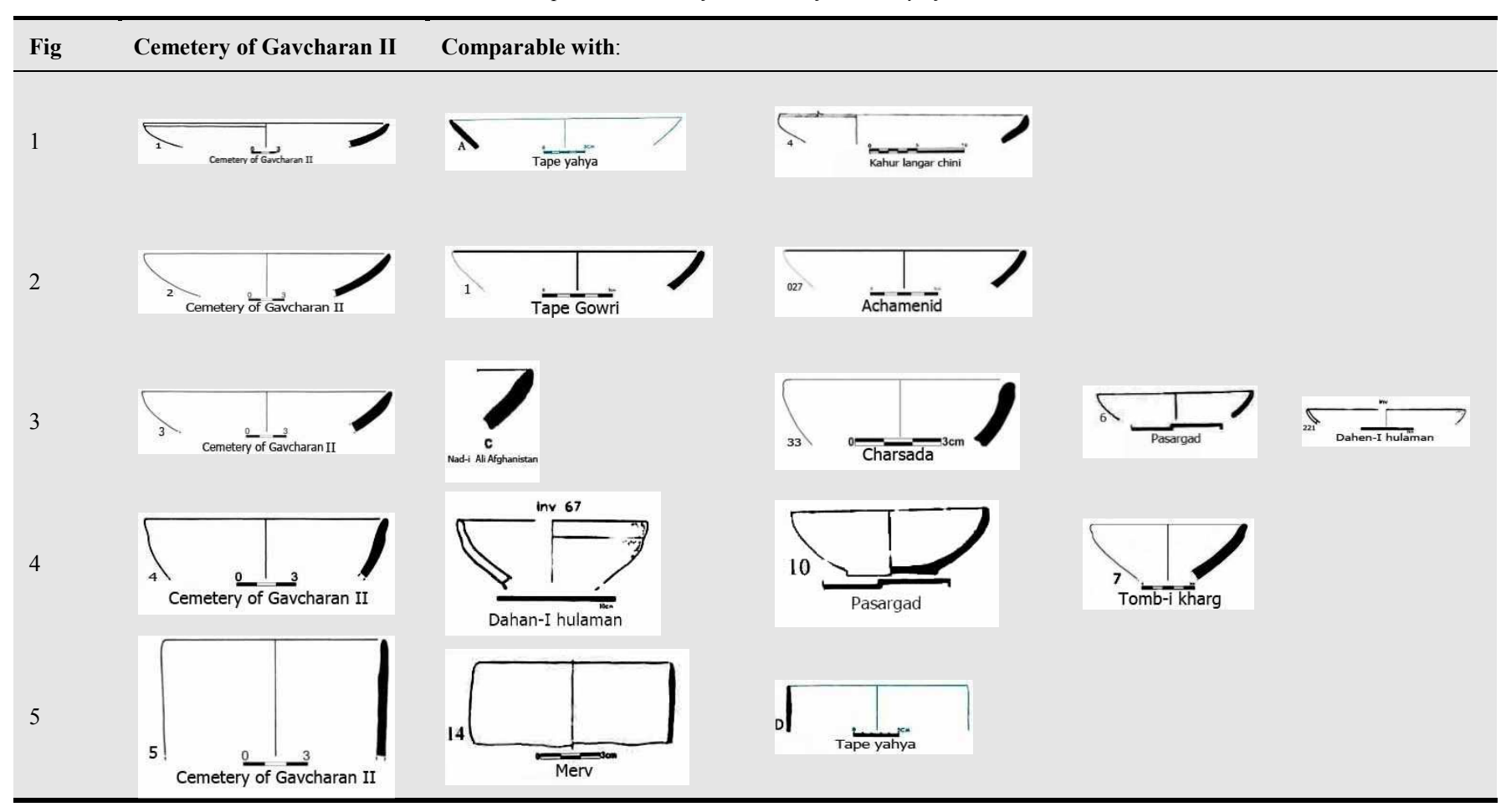

Table 6. Specification Table of pottery, Gavcharan II.

\begin{tabular}{|c|c|c|c|c|}
\hline Site & Fig & $\begin{array}{l}\text { Specification:1(Type),2.(Coloroftemper)‘3.(Temper), } \\
\text { 4.(decoration),5.(Weyofprodoction),6.(Weyoffiring),7.(slip), } \\
\text { 8.(Dia- rim),9.(Dia- base). }\end{array}$ & $\begin{array}{l}\text { Comparable } \\
\text { with: }\end{array}$ & Refrence \\
\hline \multirow{2}{*}{$\begin{array}{l}\text { Cemetery of } \\
\text { Gavcharan II }\end{array}$} & \multirow{2}{*}{1} & \multirow{2}{*}{$\begin{array}{l}\text { 1. Rim‘2.Brick-red‘3.Sand`4.(-),5.Wheel made,6.Perfect ‘7.Buff॰8 .(26) ، } \\
\text { 9.(-). }\end{array}$} & Yahya Hill & $\begin{array}{l}\text { Lamberg-Karlovsky } \\
\text { 1967-1969, } \\
\text { fig 4.9:a }\end{array}$ \\
\hline & & & $\begin{array}{l}\text { Kahur langar } \\
\text { chini }\end{array}$ & $\begin{array}{l}\text { Khosrowzadeh and Aly, } \\
\text { 1385, PL 2:4 }\end{array}$ \\
\hline \multirow{2}{*}{$\begin{array}{l}\text { Cemetery of } \\
\text { Gavcharan II }\end{array}$} & \multirow{2}{*}{2} & \multirow{2}{*}{$\begin{array}{l}\text { 1. Rim‘2.Brick-red‘3.Sand‘4.(-),5.Wheel made‘6.Perfect`7 .Buff ‘8 .(20) ، } \\
\text { 9.(-). }\end{array}$} & Tape Gowri & $\begin{array}{l}\text { Mehrafarin, 1386, Tr } \\
\text { 027:D7 }\end{array}$ \\
\hline & & & Achamenid & $\begin{array}{l}\text { Ataee, } 1383 \text {, } \\
\text { pl 3:16 }\end{array}$ \\
\hline \multirow{4}{*}{$\begin{array}{l}\text { Cemetery of } \\
\text { Gavcharan II }\end{array}$} & \multirow{4}{*}{3} & \multirow{4}{*}{ 1. Rim‘2.Buff‘3.Sand‘4.(-).5.Wheel made‘6 .Perfect ,7.Buff، 8 .(18) ،9.(-). } & $\begin{array}{l}\text { Nad-i Ali in } \\
\text { Afghanistan }\end{array}$ & $\begin{array}{l}\text { Dales } 1977, \\
\text { fig } 17: \mathrm{c}\end{array}$ \\
\hline & & & Charsada & $\begin{array}{l}\text { Wheeller } 1962 \text {, } \\
\text { fig 14: } 33\end{array}$ \\
\hline & & & Pasargad & $\begin{array}{l}\text { Stronakh, } 1379 \\
\text { ! fig } 6: 110\end{array}$ \\
\hline & & & Dahan-I Hulama & $\begin{array}{l}\text { Harrassowitz 2010, fig } \\
7: 221\end{array}$ \\
\hline \multirow{3}{*}{$\begin{array}{l}\text { Cemetery of } \\
\text { Gavcharan II }\end{array}$} & \multirow{3}{*}{4} & \multirow{3}{*}{$\begin{array}{l}\text { 1.Rim‘2.Buff‘3.Sand‘4‘(-).5. Wheel made‘6. Perfect ‘7.Buff } \\
\text { ‘8.(12) ‘9.(-). }\end{array}$} & $\begin{array}{l}\text { Dahane- } \\
\text { Gholaman }\end{array}$ & $\begin{array}{l}\text { Harrassowitz 2010, fig } \\
\text { 5: } 67\end{array}$ \\
\hline & & & Pasargad & $\begin{array}{l}\text { Stronakh, } 1379 \\
\text { :fig } 10: 110\end{array}$ \\
\hline & & & Tomb-i Kharg & $\begin{array}{l}\text { Shahsavari, } 1388 \text {, } \\
\text { pl 5-4:7 }\end{array}$ \\
\hline \multirow{2}{*}{$\begin{array}{l}\text { Cemetery of } \\
\text { Gavcharan II }\end{array}$} & \multirow{2}{*}{5} & \multirow{2}{*}{$\begin{array}{l}\text { 1. Rim‘2.Buff‘3.Gritandsand‘4.(-),5.Wheel made` } 6 \text {.Imperfect ‘7.Buff‘8 . } \\
\text { (10) ،9.(-). }\end{array}$} & Merv & $\begin{array}{l}\text { Usmanova,1992, fig } \\
7: 14\end{array}$ \\
\hline & & & Yahya Hill & $\begin{array}{l}\text { Lamberg-Karlovsky } \\
\text { 1967-1969, } \\
\text { fig 43:D }\end{array}$ \\
\hline
\end{tabular}


Table 7. Comparative Table of Potteries ofCemetery of Deh jalal mil farhad.

Fig Deh jalal mil farhad

Table 8. Specification Table of pottery, Deh jalal mil farhad.

\begin{tabular}{|c|c|c|c|c|}
\hline Site & Fig & $\begin{array}{l}\text { Specification:1(Type`2.(Coloroftemper), } \\
\text { 3.(Temper),4.(decoration), } \\
\text { 6.(Weyoffiring), 7.(slip), 8.(Dia- rim), 9.(Dia- base). }\end{array}$ & Comparable with: & Refrence \\
\hline Deh jalal mil farhad & 1 & $\begin{array}{l}\text { 1. Rim`2.Brick-red‘3.Gritandsand‘4.(-),5.Wheel } \\
\text { ،6.Perfect، 7.Brick-red,8 .(16) ‘9.(-). }\end{array}$ & $\begin{array}{l}\text { Nadali in Afghanistan } \\
\text { Hafun } \\
\text { Khorhe } \\
\text { Dahane Gholaman }\end{array}$ & $\begin{array}{l}\text { Dales, 1977, fig 17: j } \\
\text { Smith and Writh, } \\
\text { 1998. pl 3, fig } 4 \\
\text { Rahbar,1375, surface, } \\
\text { fig 8 } \\
\text { Zehbary, 1391, } \\
\text { pl 9:14 }\end{array}$ \\
\hline Deh jalal mil farhad & 2 & $\begin{array}{l}\text { 1. Rim`2. Brick-red، 3.Sand`4،(-).5. Wheel made، 6.Perfect } \\
\text { ‘7 .Brick-red ، } 8 \text {.(16) ،9.(-). }\end{array}$ & $\begin{array}{l}\text { Qaleh-i sam in the East Iran } \\
\text { Kahur langar chini } \\
\text { Baluchestan pottery }\end{array}$ & $\begin{array}{l}\text { Haerinck, 1376, pa:36, } \\
\text { fig:7 } \\
\text { Khosrowzadeh and } \\
\text { Aly, 1385, PL 2:7 } \\
\text { Alizade, 1391, } \\
\text { pl 22,fig109 }\end{array}$ \\
\hline Deh jalal mil farhad & 3 & $\begin{array}{l}\text { 1.Rim‘2.Buff‘3.Sand‘4،(-).5. Wheel made ‘6.Perfect ‘7.Buff، } \\
8 \text {.(9) ‘9.(-) }\end{array}$ & $\begin{array}{l}\text { Nkhl Ebrahimi } \\
\text { Khorhe }\end{array}$ & $\begin{array}{l}\text { Lamberg-Karlovsky } \\
\text { 1967-1969, fig 5.28:b } \\
\text { Sarlak, 1389 } \\
\text {,season4, fig:204 } \\
\text { Rahbar, 1375؛ } \\
\text { Tr 11:c8 }\end{array}$ \\
\hline
\end{tabular}

Table 9. Comparative Table of Potteries of Cemetery of Gavcharan I.

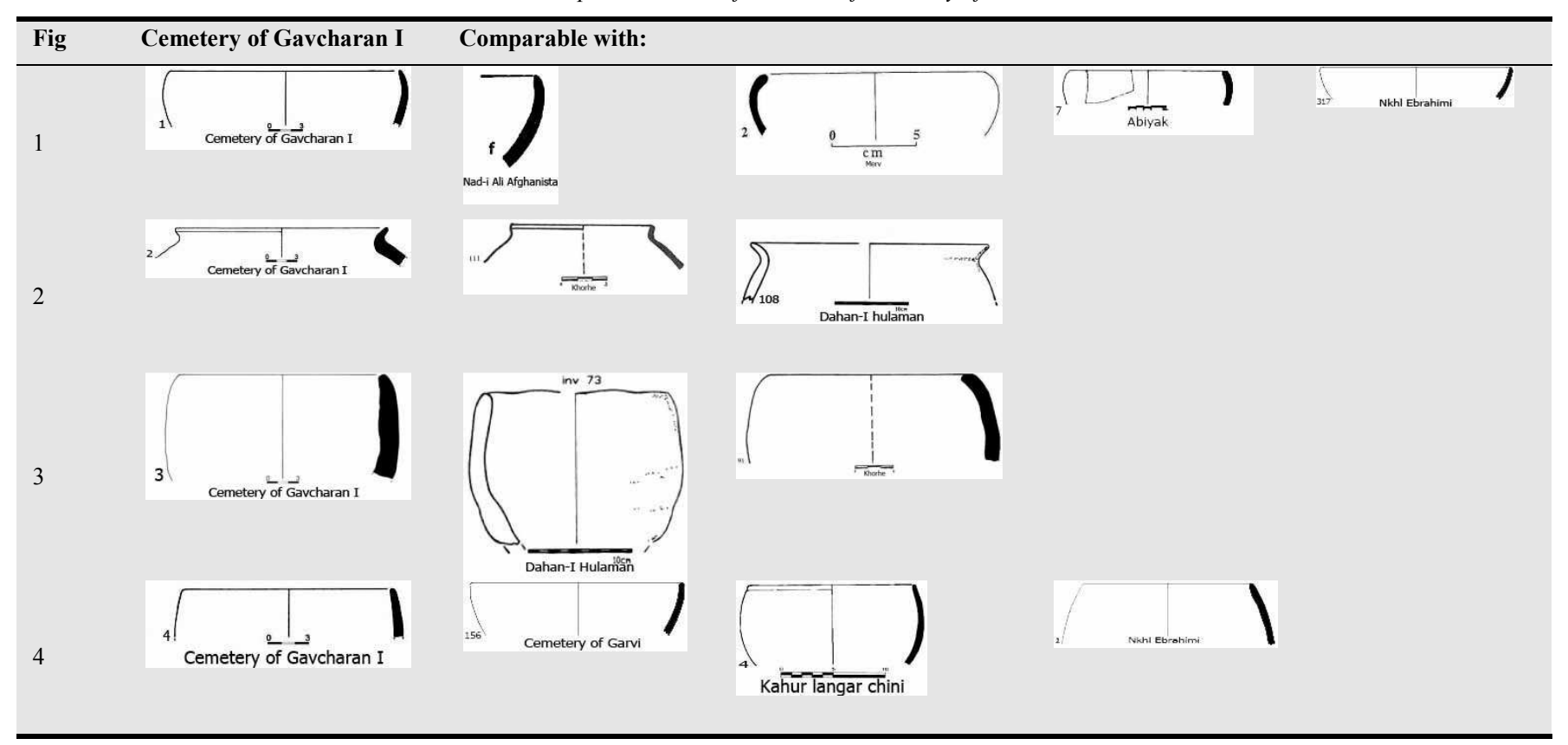


Table 10. Specification Table of pottery, Gavcharan I.

\begin{tabular}{|c|c|c|c|c|}
\hline Site & Fig & $\begin{array}{l}\text { Specification:1.(Type)‘2.(Coloroftemper‘3.(Temper), } \\
\text { 4.(decoration),5.(Weyofprodoction)‘6.(Weyoffiring), } \\
\text { 7.(slip),8.(Dia-rim),9.(Dia- base). }\end{array}$ & $\begin{array}{l}\text { Comparable } \\
\text { with: }\end{array}$ & Refrence \\
\hline \multirow[b]{3}{*}{$\begin{array}{l}\text { Cemetery of } \\
\text { Gavcharan I }\end{array}$} & \multirow[b]{3}{*}{1} & \multirow[b]{3}{*}{$\begin{array}{l}\text { 1. Rim‘2.Buff‘3.Sand and chaff‘4.(-),5.Wheel made ‘6 .Perfect • 7.Buff } 8 \text {. (20) ، } \\
\text { 9.(-). }\end{array}$} & $\begin{array}{l}\text { Nadali in } \\
\text { Afghanistan }\end{array}$ & $\begin{array}{l}\text { Dales, 1977, fig } \\
16: \mathrm{f}\end{array}$ \\
\hline & & & Merv & $\begin{array}{l}\text { Usmanova, } \\
\text { 1995, fig 11:2 }\end{array}$ \\
\hline & & & Nkhl Ebrahimi & $\begin{array}{l}\text { Najafi and } \\
\text { Makhi and } \\
\text { Akhondi, 1388, } \\
\text { pl:2, } \\
\text { right fig:7 } \\
\text { Sarlak,1389, } \\
\text { season4, } \\
\text { fig: } 317\end{array}$ \\
\hline \multirow{2}{*}{$\begin{array}{l}\text { Cemetery of } \\
\text { Gavcharan I }\end{array}$} & \multirow{2}{*}{2} & \multirow{2}{*}{ 1. Rim‘2.Buff‘3.Sand‘4.(-),5.Wheel made‘6.Perfect‘7.Buff‘8 .(20), 9.(-). } & Khorhe & $\begin{array}{l}\text { Rahbar, } 1375 \text { ؛sur } \\
\text { face, } 111\end{array}$ \\
\hline & & & $\begin{array}{l}\text { Dahan-I } \\
\text { Ghulaman }\end{array}$ & $\begin{array}{l}\text { Harrassowitz } \\
2010 \text {, fig } 4: 108\end{array}$ \\
\hline \multirow{2}{*}{$\begin{array}{l}\text { Cemetery of } \\
\text { Gavcharan I }\end{array}$} & \multirow{2}{*}{3} & \multirow{2}{*}{$\begin{array}{l}\text { 1.Rim‘2.Brick-red‘3 .Grit and sand‘4.(-),5 .Hand made‘6.Imperfect، } 7 \text {.Brick-red ، } \\
8 \text {.(19), 9.(-). }\end{array}$} & $\begin{array}{l}\text { Dahan-I } \\
\text { Ghulaman }\end{array}$ & $\begin{array}{l}\text { Harrassowitz } \\
\text { 2010, fig 1: } 73\end{array}$ \\
\hline & & & Khorhe & $\begin{array}{l}\text { Rahbar, } 1375 \\
91: \mathrm{E} 8\end{array}$ \\
\hline \multirow{3}{*}{$\begin{array}{l}\text { Cemetery of } \\
\text { Gavcharan I }\end{array}$} & \multirow{3}{*}{4} & \multirow{3}{*}{ 1. Rim‘2.Buff‘3.Sand‘4.(-),5.Wheel made‘ Perfect.6 ‘7 .Buff‘8 .(15) ‘9.(-). } & $\begin{array}{l}\text { Cemetery of } \\
\text { Garvi }\end{array}$ & $\begin{array}{l}\text { Sarlak, } 1387, \\
\text { season1, fig: } 156\end{array}$ \\
\hline & & & $\begin{array}{l}\text { Kahur langar } \\
\text { chini }\end{array}$ & $\begin{array}{l}\text { Khosrowzadeh } \\
\text { and Aly, } 1385 \text {, } \\
\text { PL 6:4 }\end{array}$ \\
\hline & & & Nkhl Ebrahimi & $\begin{array}{l}\text { Sarlak, } 1389 \text {, } \\
\text { season4, fig:2 }\end{array}$ \\
\hline
\end{tabular}

Table 11. Comparative Table of Potteries of Cemetery of Moshtin 1 Cemetery.

Fig Cemetery of moshtin I

Table 12. Specification Table of pottery, Moshtin 1 Cemetery.

\begin{tabular}{|c|c|c|c|c|}
\hline Site & Fig & $\begin{array}{l}\text { Specification:1.(Type)‘2.(Coloroftemper),3.(Temper), 4.(decoration), } \\
\text { 5.(Weyofprodoction),6.(Weyoffiring),7.(slip), 8.(Dia- rim),9.(Dia- base). }\end{array}$ & $\begin{array}{l}\text { Comparable } \\
\text { with: }\end{array}$ & Refrence \\
\hline \multirow{8}{*}{$\begin{array}{l}\text { Cemetery of } \\
\text { moshtin I }\end{array}$} & \multirow{8}{*}{1} & \multirow{8}{*}{ 1. Rim‘2.Brick-red‘3 .Sand‘4،(-).5. Wheel made‘6 .Perfect‘ 7 .Buff ‘8 . (18), 9.(-). } & $\begin{array}{l}\text { Nadali in } \\
\text { Afghanistan }\end{array}$ & $\begin{array}{l}\text { Dales 1977, } \\
\text { fig 16:c }\end{array}$ \\
\hline & & & \multirow{3}{*}{ Yahya Hill } & Lamberg- \\
\hline & & & & Karlovsky196 \\
\hline & & & & $\begin{array}{l}1-1909, \mathrm{ng} \\
4.22\end{array}$ \\
\hline & & & & $\begin{array}{l}\text { Najafi and } \\
\text { makhi and }\end{array}$ \\
\hline & & & Abiyek & akhondi, \\
\hline & & & & $\begin{array}{l}\text { 1388, pl:2, } \\
\text { Right, fig:7 }\end{array}$ \\
\hline & & & Khorhe & $\begin{array}{l}\text { Rahbar, } 1375, \\
56: c 8\end{array}$ \\
\hline
\end{tabular}

Table 13. Comparative Table of Potteries of Cemetery of Pishok 1 Cemetery.

Fig Cemetery of pishok I


Table 14. Specification Table of pottery, Pishok 1 Cemetery.

\begin{tabular}{|c|c|c|c|c|}
\hline Site & Fig & $\begin{array}{l}\text { Specification:1.(Type),2.(Coloroftemper),3.(Temper),4.(decoration),5.(Weyofprod } \\
\text { oction),6.(Weyoffiring),7.(slip),8.(Dia- rim), 9.(Dia- base). }\end{array}$ & Comparable with: & Refrence \\
\hline \multirow{4}{*}{$\begin{array}{l}\text { Cemetery of } \\
\text { pishok I }\end{array}$} & \multirow{4}{*}{1} & \multirow{4}{*}{$\begin{array}{l}\text { 1.Rim‘2.Brick-red‘3.Gret and sand‘4.(-), 5.Handmade ‘6.Imperfect‘7.Buff8,-). } \\
\text { ‘(9.(-). }\end{array}$} & $\begin{array}{l}\text { Dahane } \\
\text { Gholaman }\end{array}$ & $\begin{array}{l}\text { Harrassowitz } \\
2010 \text {, fig 4: } 28 b\end{array}$ \\
\hline & & & Khorhe & $\begin{array}{l}\text { !Rahbar, } \\
\text { 1375110:E8 }\end{array}$ \\
\hline & & & Abiyek & $\begin{array}{l}\text { Najafi and makhi } \\
\text { and akhondi, } \\
\text { 1388, pl:6,left, } \\
\text { fig:6 }\end{array}$ \\
\hline & & & Yahya Hill & $\begin{array}{l}\text { Karlovsky2004, } \\
\text { fig 5.21: c }\end{array}$ \\
\hline
\end{tabular}

\section{The Technical Properties}

Achaemenid potteries are simple and they have dough color and a buff and brick colored coverage. The clay is reinforced by a mixture of the Sand, gravel and sometimes with Herbal material. Most of the potteries are wheelwright, and have a regular shape and uniformity. Just few of them are handmade. The temperature was great to cook the potteries and their texture is rigid.

\subsection{Shapes}

The potteries are simple with various shapes such as mug, bowls and jars.

\subsection{Mugs}

This mug has an inner narrow edge. There are similar samples in Yahya Hill [19], and Dahane Gholaman [22].

\subsection{Bowl}

Hemispherical bowl with rounded edges and sometimes a narrow line can be seen on the edge. There are similar samples in Yahya Hill [19], Kahour Langar Chini, (Table 5, No 1).

Angled bowl with rounded edges and inner angled body. Their similar samples can be seen in Pasargad (Table 5, no. 4) [21]. Baluchestan (Table 7, no. 2),[24]. Choghamish[25].Boushehr and Charsardra [26], (Table 1, Nos 2, 3).

The bowl with flat outer edge and angled body is similar samples, which can be seen in Pasargad [21], and Dahane Gholaman [27], (Table 1, Nos1,2,3,6 and 8).

\subsection{Jars}

Jars are divided into two groups of neck and without neck.

The jars without neck can be seen with outer edge or flat inner edge, which its outer edge is thick. The obtained samples of Zeh-Klout, can be seen in Dahane Gholaman [27]. Nadali Afghanistan (Table, 11, no. 1),[28]. Hafoun [29], and Khourhe [30], (Table 3, No 1).

The jars without neck have outer, narrow edges. Their similar samples can be seen in Yahya Hill [19],(Table3, No 3).

\section{Parthian Era Potteries}

Ernie Herring is of researchers who studied and introduced the potteries in Parthian period. As Herring mentions, the local people made potteries and each part of Iran had different styles and properties in pottery. The most important sites are Fonouj, Hezar Mard, Damp Kouh, and Yahya Hill [31]. Most of the obtained potteries in Zeh-Klout are similar to the potteries in Yahya Hill [19]. Beside these sites, some of the Parthian era potteries in Zeh-Klout can be compared to other areas in Iran such as Khourhe [30]. Kahour Langar Chini [23], Souhar [32], Charsada [26], Hafoun (Table 7, no. 1), Marv (Table 5, no 5), and North of Oman [33]. Also there are just few obtained sites of Parthian. Their residential status is likely to be seasonal Temporary, and they were living based on the migration and nomadic traditions.

\section{Technical Properties}

The Parthian Potteries have mostly buff-colored dough and cover and also they are a mixture of Gravel, fine and medium sand, and are rarely reinforced with Herbal Materials. Most of the potteries are wheelwright, and have a regular shape and uniformity. Just few of them are handmade. The temperature was great to cook the potteries and their texture is rigid. The outer and inner levels of the potteries are mostly covered with the Grout.

\subsection{Shapes}

The Parthian Potteries in Zeh-Klout are mostly simple, without any design. They are in two groups: Bowls and Jars.

\subsection{Bowls}

The bowls with inner narrow edge and Spherical body which are similar to the potteries in Gervi [34], Marv [35], and Dezh Nakhl Ebrahimi [36],(Table9, no.4)

Bowl with outer, rounded edges and spherical body are similar to the potteries in Sam Castle [31], Kahour Langar Chini [23], and Baluchestan [24], (Table3, No2).

Bowl with inner, rounded edges and spherical body are similar to the potteries in Nada li Afghanistan[28], Yahya Hill [19], Abyek [37], Khourhe [30], and Kahour Langar Chini 
[23],(Table 9, no. 14).

\subsection{Jars}

Jars are divided into two groups of neck and without neck:

Jars with outer or vertical edge can be seen in Khourhe [30], and Dahane Gholaman [22], (Table 7, No1).
The Jar with outer, rounded edge which its outer part is thicker. The obtained sample can be seen Hafoun [29], Nad Ali Afghanistan [28], Khourhe [30] and Dahane Gholaman [27], (Table 4, No 1).

The flat edge with cylindrical body can be seen in Marve [35], and Tomb Kharg [18], (Table 3, No 5).

Table 4. Specification Table of pottery, Sar Tape Lakkoh.

\begin{tabular}{|c|c|c|c|c|}
\hline Site & Fig & $\begin{array}{l}\text { Spacification:1(Type‘2.(Coloroftemper),3.(Temper) } \\
\text { ‘4.(decoration),5.(Weyofprodoction),6.(Weyoffiring), } \\
\text { 7.(slip)‘8.(Dia- rim)،9.(Dia- base). }\end{array}$ & Comparable with: & Refrence \\
\hline Sar tape lak koh & 1 & $\begin{array}{l}\text { 1. Rim`2.Brick-red‘3 ‘Sand`.4 Black line on the edge } \\
\text { ‘5.Wheel made، 6.Perfect ‘7.Buff ‘8 (8) ‘9.(-). }\end{array}$ & $\begin{array}{l}\text { Yahya Hill } \\
\text { Zehak Sistan } \\
\text { Tomb-i Kharg }\end{array}$ & $\begin{array}{l}\text { Lamberg-Karlovsky 1972, fig 5.1:B } \\
650-500 \\
\text { ؛Mehrafarin, 1383fig 3:349 } \\
\text { ؛Shahsavari, 1388pl 5-4:13 }\end{array}$ \\
\hline Sar tape lak koh & 2 & $\begin{array}{l}\text { 1. Body-sherd`2.Brick-red`3.Sand`4 Black Geometric } \\
\text { ‘5.Wheel made‘6 .Perfect‘7.Buff‘8 ‘(-).9.(-). }\end{array}$ & 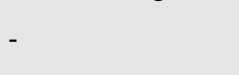 & 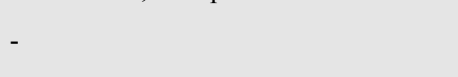 \\
\hline Sar tape lak koh & 3 & $\begin{array}{l}\text { 1. Base‘2.Brick-red‘3.Sand‘4‘(-).5. Wheel } \\
\text { made‘6.Perfect ‘7.Brick-red ‘8‘(-) . } 9.6 \text {. }\end{array}$ & $\begin{array}{l}\text { Mamasani } \\
\text { Northern Oman } \\
\text { Baluchestan pottery } \\
\text { Bandyan dargaz }\end{array}$ & $\begin{array}{l}\text { Pottsand rostaei, 2006, fig 4:112: Ts } 161 \\
\text { Decardi, 1972, fig 9:100 } \\
\text { Alizade, 1391, pl 21,fig101 } \\
\text { Rahbar, 1385؛ pl 23:3/19 }\end{array}$ \\
\hline
\end{tabular}

\section{Sassanid Era Pottery}

Louis Vandenberg collected information related to Sassanid era pottery. He believes that potteries were not considering a great attention and they were just for daily life. In general, they were such as Kitchen utensils, water jugs, vat for storing food, long and short necked decanters similar to the metal dishes and Sort of narrow containers like Current bottles. Recognizing the Parthian and Sassanid's potteries is difficult and impossible except the related layers [38]. The Sassanid potteries in this study are obtained from the residential area and based on their shapes and forms and comparing them with potteries in other sites such as Bandian Dargaz [39], and North of Oman [33], they are likely to be Sassanid era potteries.

\section{Conclusions}

Based on accomplished researches this area is suitable for human settlement based on two methods: economic production, agriculture and migration, and livestock. This cultural area has always been one of the most important commercial centers. The gateway between east and west was connected through land and sea with Central Plateau, East, North, East and South-East. Based on the studies, Ms.Choubak introduces this cultural area with Cross- cultural features. Most of the cultural findings, obtained from this area are potteries with are found in sites. For datelining these findings, we compared them with similar potteries in similar area. Most of the Zeh-Klout potteries are obtained from sites and graves which are destroyed due to the Contraband. They are created under the influence of pottery culture of other areas and they are limited in kind. The potteries in Achaemenid period are simple without any design and they are wheelwright. They are brick and buff- colored and they can be compared with the potteries from Takhte Jamshid and
Pasargadae and Dahane Gholaman in Sistan. The Parthian Potteries in Zeh-Klout are also simple and without any design with Brick and Buff-cloured dough. They are a mixture of Gravel, fine and medium sand, and are rarely reinforced with Herbal Materials. Most of the potteries are wheelwright and they have strong and rigid Dough. They are similar to the potteries in Tomb Kharag, Zahak Area, Khourhe, Nikshahr, Chabahar, Kahvar Langar Chini, Dahane Gholaman, Gervy Cemetery, Nakhle Ebrahimi, the Parthian pottery in West of Iran, Souhar, Nadali Afghanistan, North of Oman, Charsada and Marv. Due to the lack of adequate resources and few data, it seems that, the Sassanid era potteries are simple and similar to the potteries in Bandian Dargaz and North of Oman. Due to the cultural properties, the area is in relationship with India in a part and with Iranian plateau, Mesopotamiya in the other side, with Oman Sea from south and with West and Central Asia from North and North East. In different historical period, this area was the passage of many Caravans and Merchants built many castles and Caravansary in this passage.

\section{Acknowledgements}

Here the writers would like to thank Mr. Nader Ali Dadi Soleimani the manager of the archaeological site of Jiroft for his support and sincere cooperation.

\section{References}

[1] Hedaiati, H. 1339. The history of Herodotus. Translated with an introduction and explanation, University of Tehran press.

[2] Hammond, N. G. L. 1993. Sources for Alexander the Great. An analysis of Plutarchs Life and Arrians Anabasis Alexandrou. Cambridge.

[3] Chubak, H. 1383, Jazmoryan cultural continuity - the ancient city of Jiroft, Ph.D. Thesis, Tarbiat Modarres University. 
[4] Mostofi, A., 1351, "Shahdad and historical geography of Lut disert " Geographic Reporting, Vol, 8, Geographical Institute of Tehran University

[5] Beckett, P. H. T., \& Gordon, E. D. 1966. Land Use and Settlement Round Kerman in Southern Iran. Geographical Journal 132: 476-490.

[6] Stein, A. 1937. Archaeological reconnaissance in northwestern India and south-eastern Īrān. Macmillan.

[7] Harrison, J. V. 1943. The Jazmoriyan Depression, Persian Baluchestan. Geographical Journal, 101: 206-225

[8] Masumi. Gh, 1383, the history of archaeological science, Samt Publication, Tehran.

[9] Hume, G. W. (1976). The Ladizian: an industry of the Asian chopper-chopping tool complex in Iranian Baluchistan. Dorrance Publishing Company.

[10] Sajjadi, S. M. S. 1374 Eight Speech about Archeology and History of Balochestan, Cultural Heritage Organization, Tehran, first print.

[11] Chubak, H. 1381. "Conference Guide to Cultural Heritage national identity of Halil Rud to Jazmoryan", Tehran, Press Department of the introduction and training.

[12] Majid-zade. Y. 2007. Excavations at Konar Sandal: Jiroft. Campaign 2006-2007. Paper presented to the Middle Asian Intercultural Space Conference. Ravenna, 8-9 July.

[13] Dehghan , V. 1382. Survey Reports of Rudbar City, Cultural Heritage Organization of Kerman.

[14] Sheikh-Akbarizadeh, S. 1392. "The archaeological Evaluations of Zeh-klout Jazmoryan sites in the city of Rudbar " MA thesis of Archaeology, University of Kazeroon (unpublished).

[15] Boucharlat, R. and Haerinck, E. 2011. The Achaemenid Period, Encyclopedia Iranica, Ed. By Ehsan yar shater, Vol. V, Fascicle 3, central Asia XIII-CESTIYA, mazda publishers, Costa Mesa, California, PP. 302-304.

[16] Sarhaddi-Dadian, H. 2013. Archaeology of Sistan, Iran during the Achaemenian Empire (from $550 \mathrm{Bc}$ to $330 \mathrm{Bc}$ ). National University of Malaysia, Ph.d Thesis.

[17] Tohidi, F. 1382. Technology and Art of Pottery, Tehran, research organizations, and compiled books of Social Sciences (Samt).

[18] Shahsavari, M. 1388. Archaeological Systematic Review of Tamb Kharag historical hill in Rudbar, Kerman, Master Thesis, Department of Literature and Human Sciences, University of Sistan and Baluchestan.

[19] Lamberg-Karlovsy, C. C. 1967. Archeology and Metallurgical Technology in Prehistoric Afghanistan, India, and Pakistan1. American Anthropologist, 69(2): 145-162.

[20] Atai, M. T. 1383. The Introduction of the Achaemenid pottery In Fars area : a systematic review of the classification of rampart of Takhte Jamshid, master's thesis, School of Literature and Human Sciences, Tarbiat Modarres University.

[21] Stronach, D. 1978. Pasargadae: a report on the excavations conducted by the British Institute of Persian Studies from 1961 to 1963 (Vol. 1). Oxford University Press.
[22] Harrassowitz, V. 2010. Wiesbaden, Proceedings of the 6th International Congress on the Archaeology of the Ancient Near East.

[23] Khosrozadeh A. et al., 1385. Kahour Langarchi, Parthian harbor on the coast of the Persian Gulf, in: the archaeological reports (5), Institute of Archaeology, Tehran, first edition.

[24] Alizadeh, F., 1391, study of Parthian pottery samples in Baluchestan (the case of: potteries in Nikshahr and Chabahar), Master Thesis, Faculty of Literature and Human Sciences University of Sistan and Baluchestan.

[25] Delougaz, P., \& Kantor, H. J. 1996. Chogha Mish Volume 1: The First Five Seasons of Excavations 1961-1971. The University of Chicago-Oriental Institute Publications. A. Alizadeh (ed.) Vol, 101.

[26] Wheeler, M. 1962. Chārsada: A Metropolis of the North-west Frontier: Being a Report on the Excavations of 1958. Government of Pakistan and the British Academy.

[27] Zahbari. Z, Mehrafarin. R, Mousavi-Haji, S. R, Achaemenid pottery industry In Dahane Gholaman servants, MAZDAK Nameh.

[28] Dales, G. F. 1977. New Excavations at Nad-i Ali (Sorkh Dagh), Afghanistan Ed.: Center for South and Southeast Asia Studies, University of California.

[29] Smith, M. C., \& Wright, H. T. 1988. The ceramics from Ras Hafun in Somalia: notes on a classical maritime site. AZANIA Journal of the British Institute in Eastern Africa, 23(1): 115141.

[30] Rahbar, M. 1375. The Third Season of Archaeological Excavations of Khorheh, the Cultural Heritage Organization, Markazi province.

[31] Hojabri-Nobari, A., Khosrowzadeh, A., Kouhpar, S. M. M., \& Vahdatinasab, H. 2011. Trade and cultural contacts between northern and southern Persian Gulf during Parthians and Sasanians: A study based on pottery from Qeshm Island. International Journal of Humanities, 18(2), 89-115.

[32] Kervran. m, and F. Hiebert, 1991. "sohar pre-Islamic Note stratigraphique" in k.shippmann(ed.),International, Archaeology, Gulf-Archeology, Mesopotamian, Iran, Kuwait, Bahrain, Verengte Arabische Emirate and Oman, Deutsceh land, p.p 337- 348 .

[33] De Cardi, B., Vita-Finzi, C., \& Coles, A. 1975. Archaeological survey in northern Oman, 1972. East and West, 9-75.

[34] Sarlak, S. 1387. Report of the First Chapter about Exploration of Geravi cemetery 1 "Roudan city," Cultural Heritage Organization Handicrafts and Tourism Organization of Hormozgan. Bandar Abbas (unpublished).

[35] Usmanova, Z. I. 1992. New Material on Ancient Merv. Iran, 55-63.

[36] Sarlak, S. 1389. Report of the Fourth Chapter of Archaeological Excavations of Nakhl Ebrahimi area (Magi Bream) Minab city, Hormozgan province, Parse Gard magazine, the seventh year, No. 7: 28-45.

[37] Najafi, A. A., Mafi, F. and Akhoundi R. 1388. Studying the objects and the settlements of Parthian in Abyek foothills, a archaeological message, sixth year, No. XI: 78-82. 
[38] Vandenberg, Louis, 1348, archeology of ancient Iran, translation of Isa Behnam, Tehran, Tehran University.
[39] Rahbar, M. 1385. Chapter XI Investigation on Darbandian Dargaz, the country's cultural heritage. 